\title{
CCL-2 as a potential marker for remission after traumatic spinal cord injury in Chinese patients.
}

\author{
Weizhi Fang, Qixin Zheng* \\ Department of Orthopedics, Union Hospital, Tongji Medical College, Huazhong University of Science and Technology, \\ Wuhan 430022, China
}

\begin{abstract}
Objectives: To state the correlation between CCL-2, CCL-4 and CXCL-5 serum degrees and remission following traumatic spinal cord injury (TSCI) in a human protocol in comparison with animal research works.

Methods: We performed the examination of the serum levels of CCL-2, CCL-4 and CXCL-5 across an eight week duration; especially, at the time of admittance and 4, 9 and $12 \mathrm{~h}, 1$ and 3 days and 1, 2, 4, as well as 8 weeks subsequent to trauma. In accordance with the design of our research, we performed a matching 12 Chinese patients with TSCI as well as neurological remission with 12 Chinese patients having a preliminary ASIA A grade together with no neurological remission. Overall 12 patients having vertebral fracture without neurological shortages acted as control. Our analysis was carried out with the use of a Luminex Cytokine Panel.

Results: The observation received through our research exhibited dissimilarities in the serum expression layouts of CCL-2 in relation to the neurological remission (CCL-2 at admission $P=0.001$ ). Serum degrees of CCL-2 as well as CCL-4 were considerably varied in patients having or not having neurological remission.

Conclusion: As indicated by our findings, peripheral serum analysis is considered as an appropriate idea for the prediction of the patient's capacity for neurological remission subsequent to TSCI. That is why the current research work provides the introduction of a potential technique for prospective inspection of the ideas together with tracking methodologies for the prevalent therapies. As indicated by the findings, prospective examinations with an extended specimen size are required for the purpose of developing inspection, prognostic as well as scoring systems.
\end{abstract}

Keywords: Spinal cord injury, CCL-2, Inflammatory factor.

Accepted on September 11, 2019

\section{Introduction}

Spinal cord injury (SCI) continues to be one of the most demanding injuries in the contemporary medicine. SCIs together with consequent paraplegia sternly bothers patient's standard of life, in addition to giving rise to fiscal issues for not only the patient but also the associated families [1-3]. In addition to surgical treatment as well as rehabilitation, as of now, no direct treatment for traumatic SCI, for instance medication, is existent, accordingly constraining the success of accessible cures and letting an elevated threat of a discontented result [4,5]. In addition, proof in respect of the most therapeutic strategies is also very rare [6]. Till today, no authentic marker is available that is capable of indicating a promising capacity for the remission in Chinese patients. That is the reason additional information on the biochemical mechanisms is required following the SCI. The pathophysiological mechanism following the SCI is considered as intricate, together with comprising a first and second stage, which are associated with the mechanical trauma and, thereafter, an array of pathophysiological mechanisms. All through the sub-acute phase, the inflammatory feedback counts on a preliminary wave of infiltrating neutrophils, which precede a shift of monocytes as well as macrophages into the damaged area with the use of several processes.

In this research work, we aimed at determining a prognostic framework in order to evaluate the remission capacity following SCI on the bases of promising biomarkers CXCLs as well as CCLs in peripheral blood serum [7]. Because of their significance in (neuro-) inflammatory mechanisms, especially subsequent to the traumatic damages, we made a decision to perform the investigation of the serum degrees of CCL-2, CCL-4 as well as CXCL-5 [8-10]. The findings of this research aim at providing an advanced possible diagnostic approach for helping predict the potential remission following SCI, in addition to assessing the clinical therapies in respect of their achievements on remission levels [11-12].That is why this research threw focus on the following research questions: Is it probable to make prediction of the rehabilitation subsequent to 
SCI on the bases of serum cytokine expression levels? Is it likely to make use of analysis of the serum chemokine expression levels as a latent diagnostic instrument to monitor the neurological remission in prospective treatments subsequent to SCI? The feedbacks to these questions are expected to assist us in getting closer to the monitoring enhancement, together with establishing a purposeful score in the prediction of the remission following SCI.

\section{Materials and Methods}

From the year 2012 to 2016, 120 patients (93 men and 27 women) related to TSCI together with having received treatments in our hospital were added in our register in a prospective manner. Acquirement of blood specimen occurred in every scenarios featuring a time precision of $\pm 10 \%$ : 4 vials of serum (each $7.5 \mathrm{ml}$ ) were attained at 4,9 and 12 hours, 1 and 3 days as well as 1, 2, 4, and 8 weeks subsequent to SCI. Following twenty minutes coagulation, centrifugation of the blood specimens was performed at $3000 \mathrm{rpm}$, followed by aliquotion and storages at minus $80{ }^{\circ} \mathrm{C}$ until analysis.

In accordance with the corresponding status of the database, analyses of varied gatherings of serum specimens have been performed for addressing corresponding scientific problems [13-16]. Evaluation of the AIS grades occurred at the entry, in addition to, subsequent to twelve weeks as per the International Standards for Neurological Classification of SCI (ISNCSCI). The definition of the criterion regarding neurological remission was providing as favorable transformation of AIS grades within 12 weeks following trauma. All ISNCSCI inspections were carried out by the major physical therapist. Preliminary ISNCSCI inspections were carried out within the period of first 72 hours following the entry. Admissions of the patients employed for the registration were done within $2 \mathrm{~h}$ following the activation of the emergency response system. Realization of surgical therapy, thereafter, was performed within $3.5 \pm 1.6$ $\mathrm{h}$ following trauma. The GLP (Good Laboratory Practice) deliveries established the standard for all of the inspection of serum specimens. The use of Luminex Performance Human High-Sensitivity Cytokine Panels was made for the purpose of the quantitative determination of CCL-2, CCL-4 and CXCL-5 in the patients' serum specimens. Determination was carried out in accordance with the guidelines of the manufacturer (Catalog Number FCST03-04, Kit Lot Number 1420835). R\&D Systems (Minneapolis, MN, USA) delivered the kits. The corresponding analytes' attributes have been provided hereunder in sensitivity (pg ml-1) and standard curve range (pg ml-1): CCL-2 (9.9; 98-7940), CCL-4 (5.8; 261-21 150) and CXCL-5 (8.2; 50-12 140). Performance of all of the quantitative analyses was done in Union Hospital having affiliation with the Tongji Medical College by a lab personnel who wasn't aware of patients as well as medicinal information.

Approval of the recent research has been received from the ethics committee of the Huazhong University of Science and
Technology. Willful signing of the consent forms, together with their dating, was done by all of the research participants. All of the patients were able to make volunteer choices regarding quitting the research at any moment due to any issue. The patients that matched with the ruling out criteria (nontraumatic spinal cord injury, traumatic brain injury (TBI), severe abdominal trauma, traumatic amputation of extremities, coma and additional major trauma apart from the SCI) were not included without any omission. There was no patient that had been administered methylprednisolone sodium succinate (MPSS) all through the research contribution. Matching

For the purpose of eliminating the medicinal covariates on the corresponding cytokine level, cohorts of corresponding pairs were formed for the purpose of investigating the dissimilarities within their cytokine layouts. That is why we corresponded 12 patients with SCI and neurological remission (Group 1=G1) with 12 patients having a preliminary American Spinal Injury Association (ASIA) A grade together with no neurological remission (Group $0=\mathrm{G} 0$ ). In addition, 12 patients having a remote vertebral fracture with no neurological shortages acted as a control group (Group $\mathrm{C}=\mathrm{C}$ ).

Matching criteria involved the factors hereunder: age, sex, etiology and $\mathrm{AO}$ ( " Arbeitsgemeinschaft für Osteosynthesefragen") fracture classification. Provided the situation that one plus matches were able to be allocated, and then the patient having the most comparable medical status was given preference and vice versa. The consequent patients' demographics have been presented in Table 1, suggesting the AO classification of every patient in group $\mathrm{C}$. The matching mechanism was carried out with a retrospective approach on the bases of the medical data. Patient registration, specimen gathering together with analysis of CCL-2, CCL-4 and CXCL-5 were carried out prospectively.

\section{Statistical analysis}

Performance of explorative correlation analyses was done among all variables. Evaluations of the nonparametric test approaches were done for the purpose of investigating the location transfers between (Mann-Whitney U-test) and within groups at varied moments (Wilcoxon Signed Rank Test). Analysis of 2 plus sovereign specimens were carried out with the use of Kruskal-Wallis test. Evaluations of the categorical variables were performed with the use of the $\chi 2$ test.

\section{Results}

The design of the current research follows a prospective as well as explorative medical investigator research. Patients involved in this research matched out of 120 situations in our database. No randomization was observed. Criteria to perform the match-pair analysis possessed the patient's sex, age, etiology as well as AO categorization (Table 1). Documentation of the patients' demographics was performed; 
CCL-2 as a potential marker for remission after traumatic spinal cord injury in Chinese patients

analyses as well as comparisons of the groups were carried out as formerly published [15-18].

Table 1: a) Demographic and clinical characteristics of patients with TSCI and b) referring to the control group C.

\begin{tabular}{|c|c|c|c|c|}
\hline (a) Patients & Remission & No remission & $\mathrm{t} / \mathrm{x} 2$ & Comparison G1 G0 \\
\hline & (G1) & (G0) & & (P-value) \\
\hline $\mathrm{N}$ & 12 & 12 & & \\
\hline Age (years) & $43 \pm 12$ & $45 \pm 23$ & 0.267 & 0.792 \\
\hline Time accident/alarm to admission (min) & $73 \pm 22$ & $72 \pm 32$ & 0.089 & 0.93 \\
\hline \multicolumn{5}{|l|}{ Gender } \\
\hline Female & 4 & 3 & & 1.000 \\
\hline Male & 8 & 9 & & \\
\hline \multicolumn{5}{|l|}{ Etiology } \\
\hline Fall & 5 & 4 & & 1.000 \\
\hline Traffic & 7 & 8 & & \\
\hline \multicolumn{5}{|l|}{ AO classification } \\
\hline A & 7 & 8 & & 1.000 \\
\hline B & 3 & 2 & & \\
\hline C & 2 & 2 & & \\
\hline \multicolumn{5}{|l|}{$\mathrm{NLI}$} \\
\hline C & 3 & 6 & & 0.726 \\
\hline Th & 4 & 3 & & \\
\hline L & 3 & 1 & & \\
\hline None & 2 & 2 & & \\
\hline \multicolumn{5}{|l|}{ AIS at admission } \\
\hline A & 9 & 10 & & \\
\hline B & 2 & 2 & & \\
\hline C & 1 & 0 & & \\
\hline D & 0 & 0 & & \\
\hline$E$ & 0 & 0 & & \\
\hline \multicolumn{5}{|l|}{ AIS after 12 weeks } \\
\hline A & 0 & 10 & & \\
\hline$B$ & 1 & 2 & & \\
\hline C & 5 & 0 & & \\
\hline D & 6 & 0 & & \\
\hline$E$ & 0 & 0 & & \\
\hline (b) Patients & Control (C) & & & \\
\hline $\mathrm{N}$ & 12 & & & \\
\hline Age (years) & $43 \pm 12$ & & & \\
\hline
\end{tabular}




\begin{tabular}{ll}
\hline Female & 6 \\
\hline Male & 6 \\
\hline Etiology & 8 \\
\hline Fall & 4 \\
\hline Traffic & \\
\hline AO classification & 9 \\
\hline A & 3 \\
\hline B & 0 \\
\hline C & 0 \\
\hline
\end{tabular}

Age has been stated as mean years \pm s.d. Definition of neurological remission was made as enhancement in AlS. Performing the comparison of G0 and G1, analysis of P-values was conducted with the application of either the X2 test or Mann-WhitneyU-test, whereby the exhibit dissimilarities between G0 and G1.

\section{Patients' demographics}

In accordance with our developed matched-pair analysis, 36 patients were involved within this research work (13 women and 23 men) out of our current database that includes 120 scenarios, which were given admission from the year 2012 until the year 2016. The average age amounted to be $43 \pm 16$ years. The study group (Group S) comprised twenty four patients that had neurological impact of TSCI. The control group (Group C) comprised twelve patients that had received a traumatic damage with no neurological injury. In respect of group $\mathrm{S}$, there were twelve that had (Group 1 remission) and twelve that didn't have AIS damage (Group 0 no remission). The ones having no AIS injury were selected for having a preliminary AIS grade A. In C, no incisions were observed on the spinal cord. All of the medicinal attributes have been presented in Table 1.

Statistically, no dissimilarities were observed between research groups in respect of age, duration from accident/alarm to entry, period of the 1st operation, gender, etiology as well as AO categorization (termed as G1 and G0 in the following chapter). All of twenty four patient that had traumatic SCI (G0 and G1) underwent surgery (12 ventral $50.0 \% ; 12$ dorsal $50.0 \%) ; 15$ were given spondylodesis treatment $(75.0 \%)$. All of thirty six were added in the combined suffered vertebral fractures. The AIS grades at both the entry and discharge together with the NLI and the kind of paralysis were considerably varied in both G1 and G0 $(\mathrm{P}<0.05)$.

\section{CCL-2}

Within the initial twelve hours, average CCL-2 values amounted to be higher in G0 as compared with that in G1 and C. Levels of serum in G0 dropped from $699.36 \pm 104.54 \mathrm{pg} \mathrm{ml}$ -1 to $429.45 \pm 52.50 \mathrm{pg} \mathrm{ml}-14 \mathrm{~h}$ subsequent to the trauma. In respect of G1, average CCL-2 values exhibited a minimum $288.04 \pm 67.50 \mathrm{pg} \mathrm{ml}-1$ at 12 hours, together with top serum levels $456 \pm 67.50 \mathrm{pg} \mathrm{ml}-124$ hours following the trauma. Levels of serum in G1 stood lower as compared with that in G0 all through the duration, other than for the duration of top levels in G1 24 hours following the trauma. In respect of group
C, average CCL-2 levels amounted to be lower as compared with those of G0 and G1 all through the entire time period; serum levels displayed a reducing tendency from preliminary $349.20 \pm 95.80 \mathrm{pg} \mathrm{ml}-1$ to $247.13 \mathrm{pg} \mathrm{ml}-1$, 1 week subsequent to the trauma. Dissimilarities between G0 and G1 at admission $(\mathrm{P}=0.001)$ as well as 2 months $(\mathrm{P}=0.039)$ subsequent to trauma were quite considerable as their analysis was performed with the application of the Mann-Whitney U-test. In addition to that, findings of our research exhibited a considerable preliminary height of CCL-2 in G1 in comparison with that in $\mathrm{G} 0(\mathrm{P}=0.026)$.

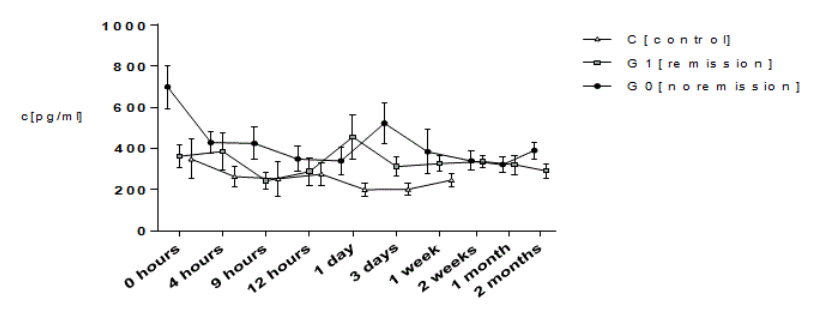

Figure 1: Serum expression patterns of G0, G1 and C expressed as means \pm s.e. of the mean and Serum level comparison of $G 0$ and $G 1$ of CCL-2 concentration. Differences were assessed with the MannWhitney U-test between both groups at each particular time point.

\section{CCL-4}

Average CCL-4 values of patients took start ranging between 108.79 and $175.95 \mathrm{pg} \mathrm{ml}-1$. That of G0 and C continued exhibiting a steady level. At a point of time of nine hours subsequent to trauma in G1, CCL-4 values showed a rise from $78.03 \pm 17.59 \mathrm{pg} \mathrm{ml}-1$ to the topmost of $214.20 \pm 96.34 \mathrm{pg} \mathrm{ml}$ -1 at twenty four hours that dropped once again to $96.18 \pm$ $19.13 \mathrm{pg} \mathrm{ml}-11$ week subsequent to trauma. Average levels of serum of G0 as well as G1 emerged falling between the ranges of 50.49 and $97.92 \mathrm{pg} \mathrm{ml-1}$ subsequent to two months. Moreover, the average levels in $\mathrm{C}$ continued to be on a steady level of $38.72 \pm 13.25 \mathrm{pg} \mathrm{ml}-11$ month subsequent to trauma. In respect of group C, average CCL-4 serum levels amounted to be lower in comparison with those of G0 and G1 always. Analyzing them using the Mann-Whitney U-test brought forth 
considerable dissimilarities nine hours $(\mathrm{P}=0.037)$ and 2 months $(\mathrm{P}=0.043)$ subsequent to trauma between $\mathrm{G} 0$ and $\mathrm{G} 1$. Average levels in G1 displayed a rise to the topmost at twenty four hours, suggesting higher levels for providing an advantageous impact on TSCI remodeling mechanisms.

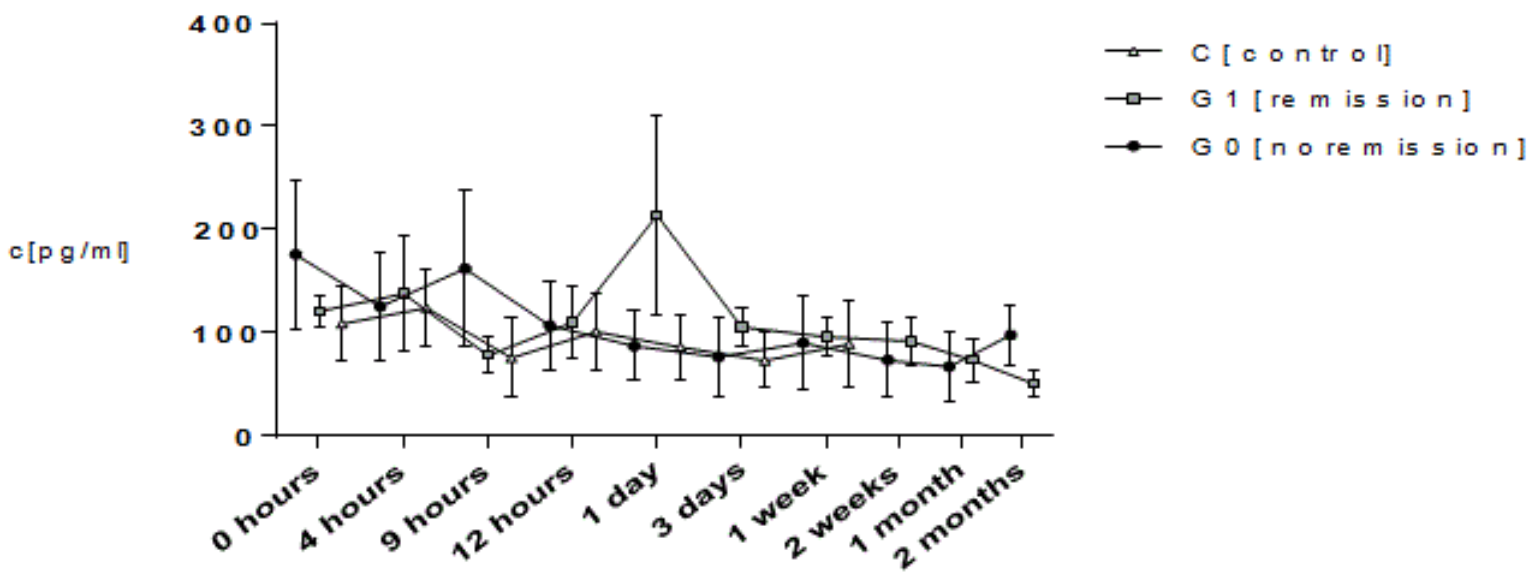

Figure 2: Serum expression patterns of G0, G1 and C expressed as means \pm s.e. of the mean and Serum level comparison of G0 and G1 of CCL-4 concentration. Differences were assessed with the Mann-Whitney U-test between both groups at each particular time point.

\section{CXCL-5}

The expression layout of average CXCL-5 values of all of the subgroups G0, G1 and C exhibited an identical layout. Taking a stat between the ranges of 713.30 and $1038.20 \mathrm{pg} \mathrm{ml}-1$, all of them touched their topmost two weeks subsequent to trauma in the ranges between 1637.15 and $1935.45 \mathrm{pg} \mathrm{ml}-1$. Ultimate average values in both $\mathrm{G} 0$ and $\mathrm{G} 1$ following two months (G1: $1252.90 \pm 201.025 \mathrm{pg} \mathrm{ml}-1 ; \mathrm{G} 0: 1112.65 \pm 392.70 \mathrm{pg} \mathrm{ml}-1)$ and in $\mathrm{C}$ following two weeks (C: $1637.15 \pm 319.73 \mathrm{pg} \mathrm{ml}-1)$ amounted to be higher in comparison with that in the start (G1: $1038.20 \pm 210.38$ pg ml-1; G0: $713.3 \pm 280.50$ pg ml-1; C: $925.65 \pm 219.73 \mathrm{pg} \mathrm{ml}-1)$. Analyzing them using the MannWhitney U-test exhibited immaterial dissimilarities between groups G0 as well as G1 always.

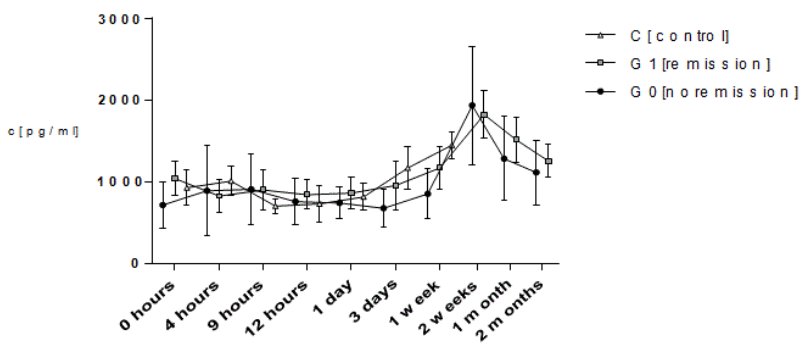

Figure 3: Serum expression patterns of G0, G1 and $C$ expressed as means \pm s.e. of the mean and Serum level comparison of G0 and G1 of CXCL-5 concentration. Differences were assessed with the MannWhitney U-test between both groups at each particular time point.

\section{Discussion}

In this research, we aimed at determining a prognostic framework in order to evaluate the remission capacity subsequent to after SCI on the bases of promising biomarkers CXCLs as well as CCLs in peripheral blood serum. The observations of our research bring forth material information regarding the preliminary evaluation of TSCI patients. As suggested by our data, dissimilarities of CCL-2 between G1 and G0 at entry $(362.13 \pm 55.55$ vs. $699.36 \pm 104.54 ; \mathrm{P}=0.001)$ as well as CCL-4 at nine hours $(78.03 \pm 17.59$ vs. $162.18 \pm$ 75.32; $\mathrm{P}=0.037$ ) imply those patients highly prone to a neurological injury within the first four hours following trauma. Moreover, the observations of our study provide the introduction of a predictive value for the distinction between patients having no probability of neurological remission and those having the elevated chances for remission.

\section{Strength and Limitations}

On the bases of future investigative research design with matched pairs, we performed the investigation of the expression layouts of CCL-2, CCL-4 as well as CXCL-5 in human peripheral serum all through the severe, subacute and mid-level stage of traumatic SCI in accordance with a trusted protocol. Our extensive database $(n=120)$ made us able to carry out a precise match-pair analysis. Despite the fact that the findings from animal frameworks are constrained by their ability to be transferred [11], yet the limitless specimen size continues to be beneficial. The current research work added a tiny group of 36 patients of matched pairs, and, that is why findings are not likely to be the representative of domestic mechanisms obviously. We made a decision for the inclusion of those patients that had varied degrees of spinal cord injury together with varied categorizations in the ASIA score in the remission cohort. In respect of the no-remission group, merely ASIA A patients was involved, doing away with the partiality of probable confusing information. Additional research works with more extended specimen size are required for addressing an inter-group (G1 vs. G0) comparison with each AISA grade, owing to the fact that the prognosis is mostly different by a 
sizeable degree. Owing to the huge standard errors of the average as well as the petite numbers of patients, the sizeable dissimilarities as stated are mandatorily required to be taken into consideration in more of a careful manner. For the purpose of further investigating the function of CCLs as well as CXCLs in the pathophysiology following TSCI, they are required to be associated with the developed inflammation markers, for instance Interferon- $\gamma$, Tumor necrosis factor- $\alpha$, Interleukin- $1 \beta$, IL-6, IL-8, IL-10 as well as vascular endothelial growth factor. In addition, we were capable of presenting discrete expression layouts in respect of both CCL-2 and CCL-4, suggesting their implication in the second stage of injury regarding G0 and G1. The two also bring forth additional information regarding the demarcation between $\mathrm{S}$ and $\mathrm{C}$.

CCL-2: CCL-2 (termed as monocyte chemoattractant protein-1 MCP-1 as well) is quite popular for being heighted in the CNS and cerebrospinal fluid of individuals having neuroinflammatory signs typified by blood-brain barrier (BBB) disruption as well as leukocyte penetration of the CNS $[19,20]$. That is why we develop a presumption that CCL-2 is to be considered as well in continued neuroinflammatory mechanisms following the traumatic SCI. In the year 2012, Roberts et al. [21] suggested that CCL-2 triggers shuttling of bcatenin between PECAM-1 (platelet endothelial cell adhesion molecule-1) as well as the adherens junction (AJ) in human brain microvascular endothelial cells in a way that the $\mathrm{AJ}$ and monolayer integrity receives transient disruption.

For the purpose of gaining more peculiar together with precise models, there is a need to perform additional research works with an extensive collective. Owing to the constrained specimen size, the current framework is likely to receive the impact from overfit. That is why we make strong recommendation to perform cross-validation on framework in prospective researches with an extensive collective for avoiding the partiality.

CCL-4: Being the major mediators of bond to the endothelial top, chemokines perform a key function in the employment of T-cell subsets (formerly confined to the CNS in vivo) across the BBB all through the CNS inflammation. Observation made by Quandt et al. [22] in the year of 2010 threw emphasis on the function and significance CNS-derived CC-chemokines for the regulation of the traffic of currently activated T-cell subsets across cytokine-activated cerebral endothelium in inflammatory ailments. They stated CCL-4 together with CCL-5 for enhancing the bond of particular CD4+ T-Cell subsets to human brain endothelial cells. There is need to perform additional research for the purpose of clarifying reasons regarding the substantial CI in G1 at twenty four hours. Latent details are likely to be attained with the help of categorization and phenotypisation of immune cells in the incision site, followed by complementation by a functional inspection. Correlations of CSF, Serum and parenchymal cytokine expression are expected to deliver further information regarding the comprehension of biochemical mechanism, together with the establishment of forecasting and inspecting systems.
CXCL-5: Despite the reports suggesting the CXCL-5 possessing neuroprotective impact subsequent to TSCI [23], yet the findings of our research throw light on no additional information in respect of neither recognition of a continued neurotrauma nor distinction between G1 and G0. CXCL-5 somewhat suggests a systemic implication in overall recovery mechanisms subsequent to trauma. Additional research, that is why, is required to throw emphasis on CXCL locally.

\section{Conclusion}

Data for systemic degrees of CCL-2 and CCL-4 extracted from peripheral serum analysis bring forth critical information regarding the biochemical mechanisms continued subsequent to TSCI. Despite the fact that critical consideration should be given to the current findings because of petite patient collective, yet they are likely to develop the foundation of prospective study dealing with the investigate as well as extrapolative scoring methodologies in TSCI with the use of research with extended specimen size.

\section{References}

1. Rowland JW, Hawryluk GW, Kwon B, Fehlings MG. Current status of acute spinal cord injury pathophysiology and emerging therapies: Promise on the horizon. Neurosurg Focus 2008; 25: E2.

2. Boakye M, Leigh BC, Skelly AC. Quality of life in persons with spinal cord injury: Comparisons with other populations. J Neurosurg Spine 2012; 17: 29-37.

3. Biglari B, Buchler A, Reitzel T, Swing T, Gerner HJ, Ferbert T, Moghaddam A. A retrospective study on flap complications after pressure ulcer surgery in spinal cordinjured patients. Spinal Cord 2014; 52: 80-83.

4. Kwon BK, Okon EB, Plunet W, Baptiste D, Fouad K, Hillyer J, Weaver LC, Fehlings MG, Tetzlaff W. A systematic review of directly applied biologic therapies for acute spinal cord injury. J Neurotrauma 2011; 28: 1589-1610.

5. Evaniew N, Belley-Cote E P, Fallah N, et al. Methylprednisolone for the treatment of patients with acute spinal cord injuries: A systematic review and metaanalysis. J Neurotrauma 2016; 33: 468-481.

6. Walters B C, Hadley M N, Hurlbert R J, et al. Guidelines for the management of acute cervical spine and spinal cord injuries: 2013 update. Neurosurgery 2013; 60: 82-91.

7. Yokobori S, Zhang Z, Moghieb A, Aarabi B, Dhall SS, Gelb DE, Harrigan MR, Rozelle CJ, Ryken TC, Theodore N. Acute diagnostic biomarkers for spinal cord injury: Review of the literature and preliminary research report. World Neurosurg 2015; 83: 867-878.

8. Tyagi P, Kadekawa K, Kashyap M, Pore S, Yoshimura N. Spontaneous recovery of reflex voiding following spinal cord injury mediated by anti-inflammatory and neuroprotective factors. Urology 2016; 88: 57-65.

9. Lee SI, Jeong SR, Kang YM, Han DH, Jin BK, Namgung U, Kim BG. Endogenous expression of interleukin-4 
regulates macrophage activation and confines cavity formation after traumatic spinal cord injury. J Neurosci Res 2010; 88: 2409-2419.

10. Brambilla R, Bracchi-Ricard V, Hu WH, Frydel B, Bramwell A, Karmally S, Green EJ, Bethea JR. Inhibition of astroglial nuclear factor kappaB reduces inflammation and improves functional recovery after spinal cord injury. $\mathrm{J}$ Exp Med 2005; 202: 145-156.

11. Kwon BK, Streijger F, Hill CE, Anderson AJ, Bacon M, Beattie MS, Blesch A, Bradbury EJ, Brown A, Bresnahan JC, Case CC, Colburn RW, David S, Fawcett JW, Ferguson AR, Fischer I, Floyd CL, Gensel JC, Houle JD, Jakeman LB, Jeffery ND, Jones LA, Kleitman N, Kocsis J, Lu P, Magnuson DS, Marsala M, Moore SW, Mothe AJ, Oudega M, Plant GW, Rabchevsky AS, Schwab JM, Silver J, Steward O, Xu XM, Guest JD, Tetzlaff W. Large animal and primate models of spinal cord injury for the testing of novel therapies. Exp Neurol 2015; 269: 154-168.

12. Kwon BK, Stammers AM, Belanger LM, Bernardo A, Chan D, Bishop CM, Slobogean GP, Zhang H, Umedaly H, Giffin M, Street J, Boyd MC, Paquette SJ, Fisher CG, Dvorak MF. Cerebrospinal fluid inflammatory cytokines and biomarkers of injury severity in acute human spinal cord injury. J Neurotrauma 2010; 27: 669-682.

13. Biglari B, Büchler A, Swing T, Biehl E, Roth HJ, Bruckner T, Schmidmaier G, Ferbert T, Gerner HJ, Moghaddam A. Increase in soluble CD95L during subacute phases after human spinal cord injury: a potential therapeutic target. Spinal Cord 2013; 51: 183-187.

14. Biglari B, Büchler A, Swing T, Child C, Biehl E, Reitzel T, Bruckner T, Ferbert T, Korff S, Rief H, Gerner HJ, Moghaddam A. Serum sCD95L concentration in patients with spinal cord injury. J Int Med Res 2015; 43: 250-256.

15. Ferbert T, Child C, Graeser V, Swing T, Akbar M, Heller R, Biglari B, Moghaddam A. Tracking spinal cord injury: Differences in cytokine expression of IGF-1, TGF- B1, and sCD951 can be measured in blood samples and correspond to neurological remission in a 12-week follow-up. J Neurotrauma 2017; 34: 607-614.

16. Moghaddam A, Heller R, Daniel V, Swing T, Akbar M, Gerner HJ, Biglari B. Exploratory study to suggest the possibility of MMP-8 and MMP-9 serum levels as early markers for remission after traumatic spinal cord injury. Spinal Cord 2017; 55: 8-15.

17. Moghaddam A, Sperl A, Heller R, Gerner HJ, Biglari B. sCD95L in serum after spinal cord injury. Spinal Cord 2016; 54: 957-960.

18. Moghaddam A, Sperl A, Heller R, Kunzmann K, Graeser V, Akbar M, Gerner HJ, Biglari B. Elevated serum insulinlike growth factor 1 levels in patients with neurological remission after traumatic spinal cord injury. PLoS One 2016; 11: e0159764.

19. Zheng Y, Morris A, Sunkara M, Layne J, Toborek M, Hennig B. Epigallocatechin-gallate stimulates NF-E2related factor and heme oxygenase-1 via caveolin-1 displacement. J Nutr Biochem 2012; 23: 163-168.

20. Paul D, Ge S, Lemire Y, Jellison ER, Serwanski DR, Ruddle NH, Pachter JS. Cell-selective knockout and 3D confocal image analysis reveals separate roles for astrocyteand endothelial-derived CCL2 in neuroinflammation. J Neuroinflammation 2014; 11: 10.

21. Roberts SA, Sterling J, Thompson C, Harris S, Mav D, Shah R, Klimczak LJ, Kryukov GV, Malc E, Mieczkowski PA, Resnick MA, Gordenin DA. Clustered mutations in yeast and in human cancers can arise from damaged long single-strand DNA regions. Mol Cell 2012; 46: 424-435.

22. Quandt D, Stech M. Molecular evolution of the trnTUGUtrnFGAA region in Bryophytes. Plant Biol (Stuttg) 2004; 6(5):545-554.

23. Tyagi AC. Partnerships for agriculture water management. Irrigation and Drainage 2016; 65: 771-772.

\section{*Correspondence to}

Qixin Zheng

Department of Orthopedics

Union Hospital, Tongji Medical College

Huazhong University of Science and Technology

Wuhan 430022

China 